\title{
Dynamic Storage Balancing in a Distributed Spatial Index
}

\author{
Cédric du Mouza \\ CNAM \\ Paris, France \\ dumouza@cnam.fr
}

\author{
Witold Litwin \\ Univ. Paris-Dauphine \\ Paris, France \\ witold.litwin@dauphine.fr
}

\author{
Philippe Rigaux \\ Univ. Paris-Dauphine \\ Paris, France \\ philippe.rigaux@dauphine.fr
}

\begin{abstract}
We propose a general framework to index very large datasets of spatial data in a distributed system. Our proposal is built on the recently proposed Scalable Distributed Rtree (SDRtree) [4] and addresses specifically the server allocation problem. In the classical situation, a new server is assigned to the network whenever a split of a full node is required. We describe a more flexible allocation protocol which allows to cope with a temporary shortage of storage resources. We analyze the cost of this protocol, describe its features, and propose practical hints to use it appropriately. Experiments are also provided and discussed.
\end{abstract}

\section{INTRODUCTION}

Spatial indexing has been studied intensively since the early works on Rtrees and Quadtree at the beginning of the 80 's. For a recent survey see [15]. It constitutes now an integrated part of most database system engines which usually adopt the simple, flexible and efficient Rtree structure (e.g., Oracle, MySQL). Recently, the advent of popular distributed systems for sharing resources across large numbers of computers has encouraged research to extend centralized indexing techniques to support queries is such contexts. However, only a few work so far has considered the extension of spatial structures and algorithms to distributed environments. Since index structures are central to efficient data retrieval, it is important to provide indexing support for distributed processing of common spatial queries.

In [4] we describe a distributed indexing framework, based on the Rtree principles, which supports all the crucial operations found in centralized systems: insertions (without duplication or clipping) and deletions; point and window queries. We measure their complexity as the number of messages exchanged between nodes, and show that this complexity is logarithmic in the number of nodes. Our struture is adapted from the centralized one by assuming that (i) no

\footnotetext{
*Work partially funded by the WISDOM project, http://wisdom.lip6.fr
}

Permission to make digital or hard copies of all or part of this work for personal or classroom use is granted without fee provided that copies are not made or distributed for profit or commercial advantage and that copies bear this notice and the full citation on the first page. To copy otherwise, to republish, to post on servers or to redistribute to lists, requires prior specific permission and/or a fee.

Copyright 200X ACM X-XXXXX-XX-X/XX/XX $\ldots \$ 5.00$. central directory is used for data addressing and (ii) nodes communicate only through point-to-point messages. These are strong but necessary assumptions which allow us to address a wide range of shared-nothing architectures. It means in particular that we aim at an even distribution of the computing and storage load over the participating servers, and avoid to rely on "super peers" or hierarchical network topologies. We propose specialized components to deal with these specific constraints. In particular each node maintains in a local cache the image of the global tree, and bases the addressing of messages on this image.

The insertion algorithm proposed in [4] requires a split operation whenever a server becomes full, and the re-assignment of half of the objects from $S$ to a newly allocated server $S^{\prime}$. This assumes that a server is available each time it is requested, which may be difficult to achieve in settings where new storage availability cannot be guaranteed. Indeed, adding storage dynamically requires time, manpower, and a network management policy under control by a reliable institution.

In the present paper we address this issue and extend the design of our SD-Rtree structure with a storage balancing method which redistributes objects from a a full server and reorganizes accordingly the distributed tree. The method allows to deal with situations where an incoming flow of insertions is sent to a network of servers that cannot, temporarily or definitely, extends itself by allocating more storage resource. In short, our contributions can be summarized as follows:

1. we introduce a $k$-NN algorithm in the SD-Rtree framework; this algorithm turns out to be useful for our goal;

2. we describe a redistribution algorithm which saves the necessity of adding systematically new servers, at the cost of additional messages;

3. we discuss and analyze the impact of storage balancing on the insertion cost, and propose a simple parameter to achieve a trade-off between a full-split policy without balancing, and a full-balancing policy without split;

4. finally we report several experiments conducted on the SD-Rtree platform.

Our proposal constitutes a flexible storage allocation method in a distributed spatial index. The insertion policy can be tuned dynamically to cope with periods of storage shortage. In such cases storage balancing should be favored for better 
space utilization, at the price of extra messages exchanges between servers. When servers availability is not a problem, storage balancing should be avoided or used very sparsely for better performances.

\section{Related work}

Until recently, most of the spatial indexing design efforts have been devoted to centralized systems [5] although, for non-spatial data, research devoted to an efficient distribution of large datasets is well-established [3, 12, 2]. Distributed index structure are either hash-based [13], or use a Distributed Hash Table (DHT) [3]. Some others are range partitioned, starting with $\mathrm{RP}^{*}$ based [12], till BATON [8] most recently. There were also proposals based on quadtrees like hQT* [10]. [11] describes an adaptive index method which offers dynamic load balancing of servers and distributed collaboration. However the structure requires a coordinator which maintains the load of each server.

The work [9] proposes an ambitious framework termed VBI. The framework is a distributed dynamic binary tree with nodes at peers. VBI seems aiming at the efficient manipulation of multi-dimensional points. Our framework rather targets the spatial (non-zero surface) objects, as Rtrees specifically. Consequently, an we enlarge a region synchronously with any insert needing it. VBI framework advocates instead the storing of the corresponding point inserts in routing nodes, as so-called discrete data. It seems an open question how far one can apply this facet of VBI to spatial objects.

The rest of the paper recalls first the structure of the SD-Rtree and its construction (Section 2). The storage balancing method is described and discussed in Section 3. Experiments are reported in Section 4 . Section 5 concludes the paper.

\section{BACKGROUND: THE SD-RTREE}

In this section we briefly describe the aspects of the SDRtree which are useful to understand our extensions. A longer presentation can be found in [4].

\subsection{Structure of the SD-Rtree}

The SD-Rtree is a binary tree mapped to a set of servers. It is conceptually similar to the classical AVL tree, although the data organization principles are taken from the Rtree spatial containment relationship. Each internal node, or routing node, refers to exactly two children whose heights differ by at most one. This ensures that the height of a SD-Rtree is logarithmic in the number of servers. A routing node maintains also left and right directory rectangles $(\mathrm{dr})$ which are the minimal bounding boxes of, respectively, the left and right subtrees. Finally each leaf node, or data node, stores a subset of the indexed objects.

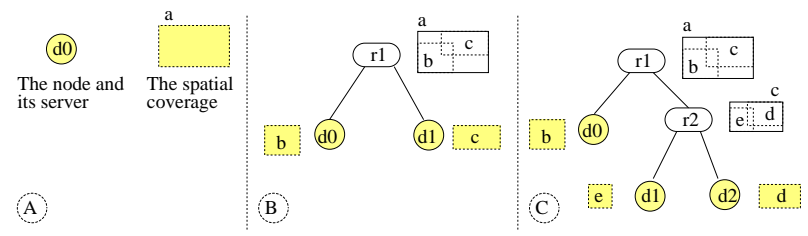

Figure 1: Basic features of the SD-Rtree
Figure 1 shows an example with three successive evolutions. Initially (part A) there is one data node $d_{0}$ stored on server 0. After the first split (part B), a new server $S_{1}$ stores the pair $\left(r_{1}, d_{1}\right)$ where $r_{1}$ is a routing node and $d_{1}$ a data node. The objects have been distributed among the two servers and the tree $r_{1}\left(d_{0}, d_{1}\right)$ follows the classical Rtree organization based on rectangle containment. The directory rectangle of $r_{1}$ is a, and the directory rectangles of $d_{0}$ and $d_{1}$ are respectively $\mathrm{b}$ and $\mathrm{c}$, with $\mathrm{a}=m b b(\mathrm{~b} \cup \mathrm{c})$. The rectangles $\mathrm{a}, \mathrm{b}$ and $\mathrm{c}$ are kept on $r_{1}$ in order to guide insert and search operations. If the server $S_{1}$ must split in turn, its directory rectangle $\mathrm{c}$ is further divided and the objects distributed among $S_{1}$ and a new server $S_{2}$ which stores a new routing node $r_{2}$ and a new data node $d_{2} . r_{2}$ keeps its directory rectangle $\mathrm{c}$ and the $\mathrm{dr}$ of its left and right children, $\mathrm{d}$ and $\mathrm{e}$, with $\mathrm{c}=m b b(\mathrm{~d} \cup \mathrm{e})$. Each directory rectangle of a node is therefore represented exactly twice: on the node, and on its parent.

A routing node maintains the id of its parent node, and links to its left and right children. A link is a quadruplet (id, dr, height, type), where id is the id of the server that stores the referenced node, $d r$ is the directory rectangle of the referenced node, height is the height of the subtree rooted at the referenced node and type is either data or routing. Whenever the type of a link is data, it refers to the data node stored on server id, else it refers to the routing node. Note that a node can be identified by its type (data or routing) together with the id of the server where it resides. When no ambiguity arises, we will blur the distinction between a node id and its server id.

The description of a routing node is as follows:

\section{Type: RoutingNode}

height, dr: description of the routing node

left, right: links to the left and right children

parent_id: id of the parent routing node

OC: the overlapping coverage

The routing node provides an exact local description of the tree. In particular the directory rectangle is always the geometric union of left.dr and right.dr, and the height is Max(left.height, right.height) +1 . OC, the overlapping coverage, to be described next, is an array that contains the part of the directory rectangle shared with other servers. The type of a data node is as follows:

Type: DataNode

data: the local dataset

$\mathrm{dr}$ : the directory rectangle

parent_id: id of the parent routing node

OC: the overlapping coverage

\subsection{The image}

An important concern when designing a distributed tree is the load of the servers that store the routing nodes located at or near the root. These servers are likely to receive proportionately much more messages. In the worst case all the messages must be first routed to the root. This is unacceptable in a scalable data structure which must distribute evenly the work over all the servers.

An application that accesses an SD-Rtree maintains an image of the distributed tree. This image provides a view which may be partial and/or outdated. During an insertion, the user/application estimates from its image the address of 
the target server which is the most likely to store the object. If the image is obsolete, the insertion can be routed to an incorrect server. The structure delivers then the insertion to the correct server using its actual routing node at the servers. The correct server sends back an image adjustment message (IAM) to the requester.

\subsection{Overlapping coverage}

Our search operations attempt to find directly, without requiring a top-down traversal, a data node $d$ whose directory rectangle $d r$ satisfies the search predicate. However this strategy is not sufficient with spatial structures that permit overlapping, because $d$ does not contain all the objects covered by $d r$. We must therefore be able to forward the query to all the servers that potentially match the search predicate. This requires the distributed maintenance of some redundant information regarding the parts of the indexed area shared by several nodes, called overlapping coverage (OC) in the present paper. So each data node $N$ maintains all the directory rectangle of the sibling of its ancestors $N_{i}$ (called in the following outer nodes) on the path from $N$ to the root of the tree. This information is stored as an array of the form $\left[1: o c_{1}, 2: o c_{2}, \cdots, n: o c_{n}\right]$, such that $o c_{i}$ is $\operatorname{outer}_{N}\left(N_{i}\right) \cdot d r$. Note that we have to trigger a maintenance operation only when this overlapping changes.

\subsection{Insertion}

We now describe insertions in the distributed tree. Recall that all these operations rely on an image of the structure (see above) which helps to remain as much as possible near the leaves level in the tree, thereby avoiding root overloading. Moreover, as a side effect of these operations, the image is adjusted through IAMs to better reflect the current state of the structure.

Assume that a client application $C$ requires the insertion of an object $o$ with rectangle $m b b$ in the distributed tree. $C$ searches its local image $I$ and determines from the links in $I$ a data node which can store $o$ without any enlargement. If no such node exist, the link whose $\mathrm{dr}$ is the closest to $\mathrm{mbb}$ is chosen ${ }^{1}$. If the selected link is of type data, $C$ addresses a message InSERT-IN-LEAF to $S$; else the link refers to a routing node and $C$ sends a message INSERT-IN-SubTREE to $S$.

- (InsERT-In-LEAF message) $S$ receives the message; if the directory rectangle of its data node $d_{S}$ covers actually $o . m b b, S$ can take the decision to insert $o$ in its local repository; there is no need to make any other modification in the distributed tree (if no split occurs); else the message is out of range, and a message INSERTIN-SubTREE is routed to the parent $S^{\prime}$ of $d_{S}$;

- (Insert-In-Subtree message) when a server $S^{\prime}$ receives such a message, it first consults its routing node $r_{S^{\prime}}$ to check whether its directory rectangle covers $o$; if no the message is forwarded to the parent until a satisfying subtree is found (in the worst case one reaches the root); if yes the insertion is carried out from $r_{S^{\prime}}$ using the classical Rtree top-down insertion algorithm. During the top-down traversal, the directory rectangles of the routing nodes may have to be enlarged.

\footnotetext{
${ }^{1}$ The image is initially empty. $C$ must know at least one
} node $N$, and send the insertion request to $N$.
If the insertion could not be performed in one hop, the server that finally inserts $o$ sends an acknowledgment to $C$, along with an IAM containing all the links collected from the visited servers. $C$ can then refresh its image.

The insertion process is shown on Figure 2. The client chooses to send the insertion message to $S_{2}$. Assume that $S_{2}$ cannot make the decision to insert $o$, because $o . m b b$ is not contained in $d_{2} . d r$. Then $S_{2}$ initiates a bottom-up traversal of the SD-Rtree until a routing node whose $\mathrm{dr}$ covers $o$ is found (node $\mathrm{c}$ on the figure). A classical insertion algorithm is performed on the subtree rooted at c. The out-of-range path (ORP) consists of all the servers involved in this chain of messages. Their routing and data links constitute the IAM which is sent back to $C$.

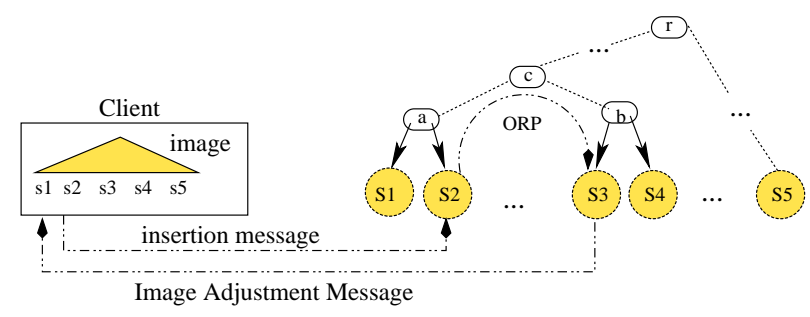

Figure 2: The insertion algorithm

In the worst case an insertion $\operatorname{costs} O(3 \log N)$ message. However, if the image is reasonably accurate, the insertion is routed to the part of the tree which should host the inserted object, and this results in a short out-of-range path with few messages. As shown by the experiments reported in [4], this strategy reduces drastically the workload of the root node.

\subsection{Node splitting}

When a server $S$ is overloaded by new insertions in its data repository, a split must be carried out. A new server $S^{\prime}$ is added to the system, and the data stored on $S$ is divided in two approximately equal subsets using a split algorithm similar to that of the classical Rtree [7] One subset is moved to the data repository of $S^{\prime}$. A new routing node $r_{S^{\prime}}$ is stored on $S^{\prime}$ and becomes the immediate parent of the data nodes respectively stored on $S$ and $S^{\prime}$.

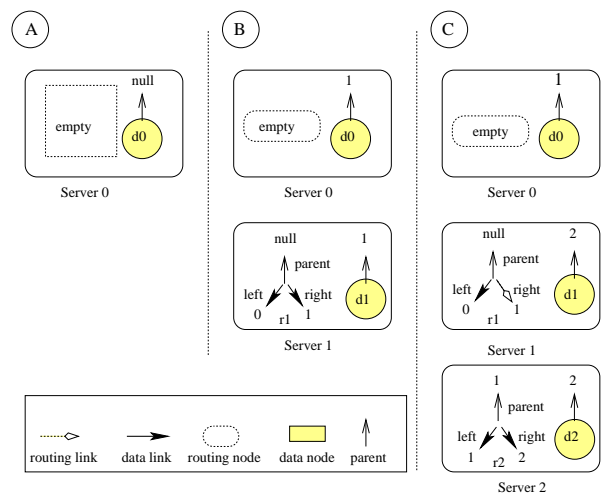

Figure 3: Split operations

An example of management and distribution of routing and data nodes is detailed on Figure 3. Initially (part A), the system consists of a single server, with id 0. Every insertion is routed to this server, until its capacity is exceeded. 
After the first split (part B), the routing node $r_{1}$, stored on server 1, keeps the following information (we ignore the management of the overlapping coverage for the time being):

- the left and right fields; both are data links that reference respectively servers 0 and 1 ,

- its height (equal to 1) and its directory rectangle (equal to mbb(left.dr, right.dr)),

- the parent id of the data nodes 0 and 1 is 1 , the id of the server that host their common parent routing node.

Since both the left and right links are of type data links, the referenced servers are accessed as data nodes (leaves) during a tree traversal.

Continuing with the same example, insertions are now routed either to server 0 or to server 1 , using a Rtree-like ChooseSubtree procedure [7, 1]. When the server 1 becomes full again, the split generates a new routing node $r_{2}$ on the server 2, with its left and right data links pointing respectively to server 1 and to server 2 , and its parent_id field referring to server 1 . At this point the tree is still balanced. It may happen that splits make the tree imbalanced. In [4] we describe a rotation mechanism to preserve the balance of the tree at a very low extra-cost.

\section{STORAGE BALANCING}

The insertion algorithm described above requires a split each time a server is full. This adds systematically a new server to the network. The institution that manages the network must be ready to allocate resource at any moment (in the case of a cluster of servers), or a free server has to be available (in the case of an unsupervised network). We present in this section a storage balancing scheme which allows a full node to transfer part of its data to lightly loaded servers whenever new storage resources cannot be allocated.

\section{$3.1 k$-NN queries}

Our technique is partially based on a $k$-NN querying algorithm decomposed in two steps:

1. find a data node $N$ that contains $P$ and evaluate locally the $k$-NN query;

2. from $N$, explore all the other data nodes which potentially contain an object closer to $P$ than those found locally.

The first step is a simple point-query. Assume that a data node $N$ containing $P$ is found. The $k$-NN query is evaluated locally, i.e., without any extra-message, using a classical $k$ closest neighbors algorithm [14]. The initial list of neighbors, found locally, is stored into an ordered list neighbors $(P, k)$.

Obviously, some closest neighbors may also be located in other nodes, which may lead to an update of neighbor $(P, k)$. In order to determine which servers must be contacted, the following strategies can be considered.

\section{Range querying}

The first approach performs a range query over the tree, the range $r$ being the distance between $P$ and the farthest object in neighbors $(P, k)$. This is illustrated on Figure 4 . The local search in node $N$ finds a set of neighbors, the

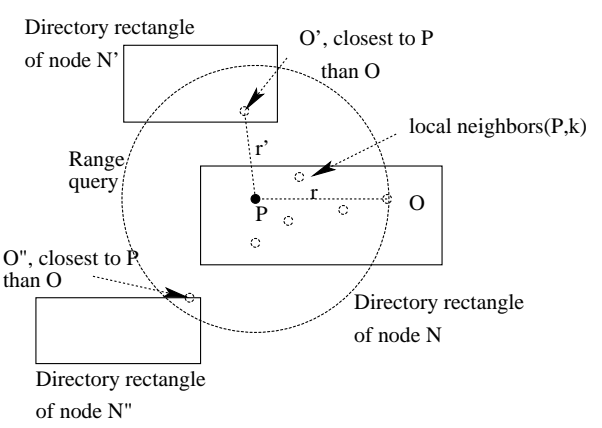

Figure 4: Range query to retrieve objects from other nodes

farthest being object $O$. The distance from $P$ to $O$ determines the range of the query.

Now, still referring to figure 4 , nodes $N^{\prime}$ and $N^{\prime \prime}$ fall into this range. Thus $N$ sends to $N^{\prime}$ and $N^{\prime \prime}$ a query with $P$ and the radius $r$. The contacted nodes send back to $N$ the object(s) that can replace one or many element(s) of neighbors $(P, k)$. For example object $O^{\prime}$, found in the space covered by $N^{\prime}$ turns out to be closer to $P$ than $O . N^{\prime}$ searches its local space for all objects whose distance to $P$ is less than $r$. Object $O^{\prime}$ is found and returned to $N$. The same process holds for $N^{\prime \prime}$, which returns object $O^{\prime \prime}$. Given the list of all objects retrieved through this process, server $N$ determines the final list of nearest neighbors. Still referring to figure 4 , and assuming $k=5$, the final list contains $O^{\prime}$ but neither $O$ nor $O^{\prime \prime}$.

\section{Improved $k$-NN search}

The above algorithm broadcasts the query to all the nodes that may potentially improve the $k \mathrm{NN}$ list. However some messages tend to be useless, as illustrated on Figure 5. Assume node $N$ is contacted first. It sends back to $N$ object $O^{\prime}$ which improves the nearest neighbors list, and reduces the maximal radius to $r^{\prime}$. Let $r^{\prime \prime}$ be the distance between $P$ and the directory rectangle of $N^{\prime \prime}$. Since $r^{\prime \prime}>r^{\prime}$, there is clearly no hope to get any closer neighbor from $N^{\prime \prime}$.

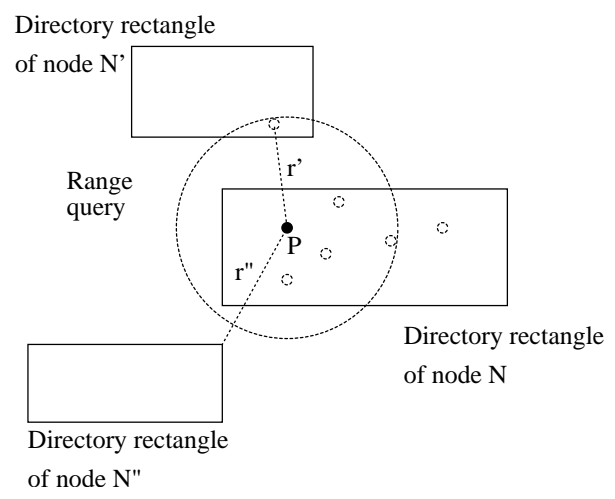

Figure 5: Improved $k-\mathrm{NN}$ query

The improved strategy, presented below, generates a chain of messages which contact the nodes in an order which is estimated to deliver the quickest convergence towards the final result. This is likely to limit the number of servers to contact. We first recall some useful distance measures for 
$k$-NN queries, as proposed in [14] and illustrated in Figure 6.

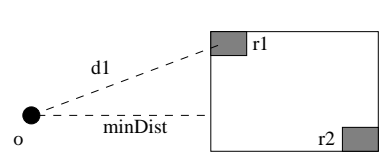

(a) first extreme case

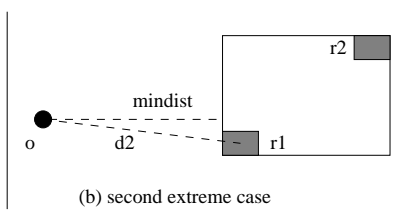

(b) second extreme case

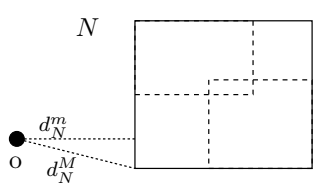

a) $N$ is visited, $d_{N}^{m}$ and $d_{N}^{M}$ are removed from lists

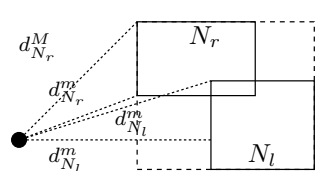

b) new distances for $N_{r}$ and $N_{l}$ inserted in lists
Figure 6: The minMaxDist

Let $R$ be the bounding box of a set of rectangles. First let minDist denote the minimal distance between a point $P$ and $R$. Second note that each of $R$ 's edges shares at least one point with one of its inner rectangles. Figure 6 shows the two extreme positions for a rectangle $r_{1}$ inside $R$ with respect to the edge closest to $P$. The maximal distance among $d_{1}$ and $d_{2}$ represents the minimal distance from $P$ to a rectangle contained in $R$. This distance is denoted $\operatorname{minMax} \operatorname{Dist}(P, R)$.

Now, given a list neighbor $s(P, k)$, let $d_{\max }$ be the distance between $P$ and the last (farthest) object in neighbors $(P, k)$. Recall from Section 2 that the overlapping coverage (OC) is an array of the form $\left[1: o c_{1}, 2: o c_{2}, \cdots, n: o c_{n}\right]$, such that $o c_{i}$ is outer $_{N}\left(N_{i}\right) . d r$. If $N^{\prime}$ is a node in the overlapping coverage of $S$, the object closest to $P$ in $N^{\prime}$ is at best at distance $\min \operatorname{Dist}(P, N . d r)$, and at worse at distance $\operatorname{minMax} \operatorname{Dist}(P, N . d r)$. Therefore if $d_{\max }<\min \operatorname{Dist}(P, N . d r)$, there is no hope to improve the current list of neighbors by contacting $N^{\prime}$.

When processing a $k$-NN query, we compute the minDist and minMaxDist distances from $P$ to all the mbb of the nodes in the OC list. We build a list $L_{m d}\left(\right.$ resp. $\left.L_{m m d}\right)$ of pairs $\left(i d_{i}, d_{i}\right)$ where $i d_{i}$ is the id of the node $N_{i}$ and $d_{i}$ the value of $\min \operatorname{Dist}\left(P, N_{i} . d r\right)$ (resp. $\operatorname{minMax} \operatorname{Dist}\left(P, N_{i} . d r\right)$ ). $L_{m d}$ and $L_{m m d}$ are sorted on $d_{i}$ in ascending order. To determine which server must be contacted we simply compare the distance from $P$ to the farthest object in neighbors $(P, k)$, denoted $d_{\max }$, and the distances in $L_{m d}$ and $L_{m m d}$. Our algorithm relies on the two following observations:

i) if $d_{\max }<\min \operatorname{Dist}\left(P, N_{i} . d r\right)$ we do not have to visit node $N_{i}$;

ii) if $\operatorname{minMax} \operatorname{Dist}\left(P, N_{i} . d r\right)<d_{\max }$ we must visit node $N_{i}$ because it contains an object closer to $P$ that the farthest object from neighbors $(P, k)$.

The algorithm maintains an ordered list of the nodes that need to be visited. The first node of the list is then contacted. If it is a data node, a local search is carried out, which possibly modifies neighbor $(P, k)$, as well as the lists $L_{m d}$ and $L_{m m d}$. If it is a routing node, it computes for its two children the distances minDist and minMaxDist, and updates $L_{m d}$ and $L_{m m d}$ accordingly. This is illustrated in figure 7 where the routing node $N$ has to be visited, regarding either its $\operatorname{minMaxDist} d_{N}^{M}$ or its minDist $d_{N}^{m}$ with query point $o$. When $N$ is contacted, it removes first $d_{N}^{M}$ $\left(\right.$ resp. $\left.d_{N}^{m}\right)$ from the list $L_{m m d}$ (resp. $\left.L_{m d}\right)$. Since $N$ knows the mbb of its two children, $N_{r}$ and $N_{l}$, it is able to compute the $\operatorname{minMaxDist} d_{N_{r}}^{M}$ (resp. $d_{N_{l}}^{M}$ ) and minDist $d_{N_{r}}^{m}$ (resp. $d_{N_{l}}^{m}$ ) for $N_{r}$ (resp. $N_{l}$ ). It inserts each of these distances in the appropriate ordered list $\left(L_{m m d}\right.$ or $\left.L_{m d}\right)$ and visits w.r.t. these lists the following node.
Figure 7: Updating $L_{m m d}$ and $L_{m d}$

In both cases, the next server in the list is contacted in turn, until no possible improvement of the nearest neighbors can be obtained. The message transmitted from one server to another contains $P$, the list neighbor $(P, k)$ at the current step of the algorithm, and the lists $L_{m d}$ and $L_{m m d}$.

An heuristic consists in contacting first the server with the minimal minMaxDist since we know for sure that this modifies neighbor $(P, k)$ and reduces $d_{\max }$. When all the servers have been explored with respect to minMaxDist, the remaining ones are contacted with respect to minDist.

The cost of this $k$-NN algorithm is in the worst case that of the simple range query approach. However in the average case the number of message may be drastically reduced.

\subsection{Revisited insertion}

Assume that an object $o$ must be inserted in a full node $N$. $N$ may require a redistribution balancing as follows. First it contacts, by sending bottom-up messages, its nearest ancestor $N_{P}$ which has at least one non-full descendant, called the pivot node. If no such ancestor is found, the tree is full. We must then proceed to a node split of $N$, as presented previously. Otherwise, since $N_{P}$ is the nearest non-full ancestor, one of its two subtrees (say, $L$ ) contains only full nodes, including $N$. The other subtree (say, $R$ ) contains at least one non-full node (Figure 8).

$N_{P}$ must move some objects from $L$ rooted at $N_{L}$ to $R$ rooted at $N_{R}$. For simplicity we describe the technique for a single object but it may be extended for redistributing larger sets.

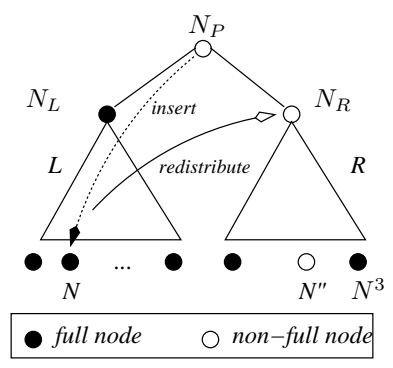

Figure 8: Overview of the load balancing strategy

We need additional information to detect the pivot node. We use two flags, Full left and Full right $_{\text {, }}$, one for each child, added to the routing node type. Flag Full $_{i}$ is set to 1 when the subtree rooted at child $i$ is full. Flag values are maintained as follows. When a leaf becomes full, a message is sent to the server that hosts its parent node to set the corresponding flag to 1 . This one may in turn, if its companion flag is already set to 1 , alert its parent, and so on, possibly up to the root, i.e., $\log (n)$ messages (effectively, quite unfre- 
quent since this implies a full tree). The flags are maintained similarly for deletions.

In order to minimize the overlap between the mbb of $L$ and $R$, we need to redistribute the object indexed by $L$ which is the closest to $R$. This can be achieved with a 1-NN query in $L$.

The query point $P$ is the centroid of the directory rectangle of $N_{R}$. With that choice the query will return an object producing a small enlargement of the overlapping coverage. The 1-NN algorithm is then initiated by $N_{R}$, which sets initially $L_{m m d}$ and $L_{m d}$ to $N_{L}$, and neighbors $(P, 1)$ to $\emptyset . N_{R}$ re-inserts then the object obtained as the result of this query in its subtree. This redistribution impacts the two subtrees $N_{L}$ and $N_{R}$ in two ways.

First, the reinserted object may have to be assigned to a full data node in the subtree rooted at $N_{R}$. This triggers another redistribution. However, since $N_{R}$ was marked as non-full, we know that the pivot node will be found in the subtree rooted at $N_{R}$. Consider for instance Figure 8 , and assume that a reinserted object must be put in the full node $N^{3}$. Since there exists at least one non-full node in $N_{R}\left(N^{\prime \prime}\right.$ for our example), the pivot node has to be a descendant of $N_{R}$.

Second the node $N$ where the initial object $o$ had to be put may become full in turn. So the redistribution algorithm has to be iterated. For the very same reasons, we know that the pivot node has to be a descendant of $N_{L}$. The load balancing stops when the chain of redistribution stops.

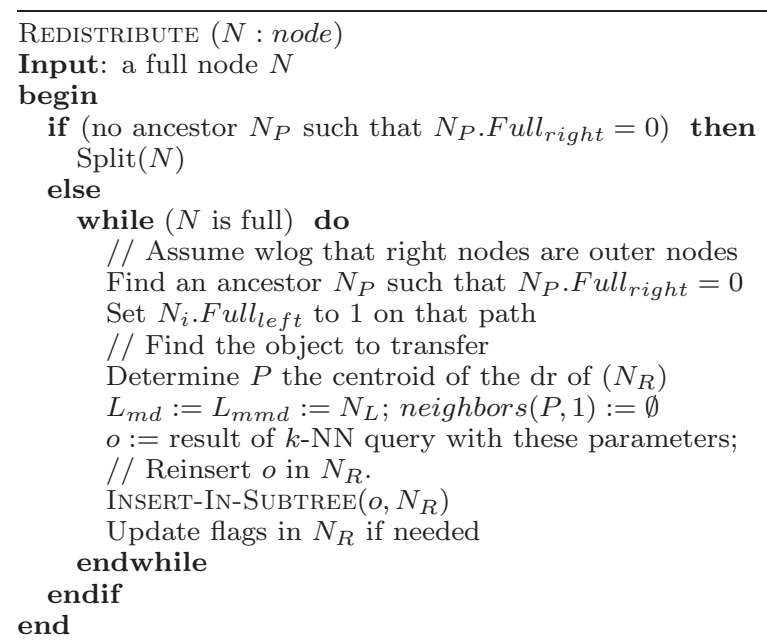

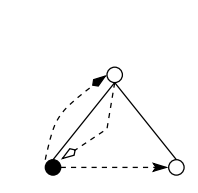

(a) $\mathrm{H}=1$

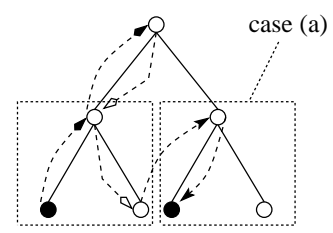

(b) $\mathrm{H}=2$

\begin{tabular}{|lll|}
\hline $\begin{array}{l}\text { pivot detection } \\
\text { message }\end{array}$ & $\begin{array}{l}1-\mathrm{NN} \text { query } \\
\text { message }\end{array}$ & reinsert \\
message
\end{tabular}

Figure 9: Redistributing data

Figure 9 illustrates the algorithm when the height of the pivot node is respectively 1 (a) and 2 (b). The algorithm analysis is as follows. When a node $N$ gets full, we need $H$ messages to find the pivot node $N_{P}$, where $H$ is the height of $N_{P}$. Then we need $H$ messages to find the object that must be moved from the left subtree in $N_{P}, N_{L}$, to the right one, $N_{R}$. The reinsertion of an object costs $H$ messages. After this first step, we possibly have to iterate the algorithm for the two insertions, respectively in $N_{L}$ and $N_{R}$ (see Figure 9 for the example with $H=2$ ). In both cases the pivot node's height is at worse $H-1$. This yields the recursive formula: $\operatorname{cost}(H)=3 H+2 \times \operatorname{cost}(H-1)$. So finally, $\operatorname{cost}(H)=$ $\sum_{i=0}^{H-1} 2^{i} \times 3(H-i)=O\left(H .2^{H}\right)$. In the worst case, $H=$ $\log _{2} n$ (i.e., the pivot node is the root) and this results in $O\left(n \log _{2} n\right)$ messages, where $n$ is the number of servers.

\subsection{Discussion}

The redistribution is costly, its main advantage being to save some splits, and thus the necessity to add servers to the structure. Note that we may simply not have the choice, if no storage resource is available! In general, we can choose between two extreme strategies: one that performs systematically a split (and adds a new server) and one that delays any split until all the servers are full. This latter clearly requires a lot of exchanges, whereas the former is not always possible. It seems convenient to provide a mean to adopt a trade-off between splits and redistributions.

The basic idea is to proceed only to "local" reorganization by limiting data redistribution to the close siblings of a full node, and not to the whole structure. As shown by the above analysis, the redistribution cost is exponential in the height of the pivot node. By bounding this height, we limit the cost of redistribution at the price or more frequent splits, and less effective storage utilization. Let $\nu$ represent the maximal height for a pivot node. The $\operatorname{Redistribute}(N)$ algorithm is simply modified as follows:

- if height $\left(N_{P}\right)>\nu$ then split $N$

- else, apply Redistribute $(N)$

The choice $\nu=0$ corresponds to a strategy where we split whenever a node is full, without any data redistribution. This minimizes the number of messages exchanged. An opposite choice is to set $\nu$ to $\infty$, allowing to choose any ancestor of a full node as a pivot, including the root, which results in a likely perfect storage utilization, but to a maximal number of messages, since a split occurs only when all the servers are full.

Parameter $\nu$ can easily be changed dynamically, which makes possible to adapt the behavior of the distributed tree to specific circumstances (i.e., a highly loaded period). Assume for instance that an initial pool of $m$ servers is available. $\nu$ can be set to 0 , thereby minimizing the number of network exchanges. When the $m$ servers are in use, and a new one is required by a split, storage balancing can be enabled by setting $\nu$ to 1 . Depending on the time necessary to allocate new servers to the pool, $\nu$ can be progressively raised, as the servers get full, until new storage space becomes available. Then it can be set to 0 again to disable the storage balancing option. So $\nu$ is a tuning parameter that brings flexibility since it allows to adapt the behavior of the system with respect to the available resources.

\section{EXPERIMENTAL VALIDATION}


We report several experiments that evaluate the performance of our proposed architecture over large datasets of 2dimensional rectangles, using a distributed structure simulator written in C. Our datasets include both data produced by the GSTD generator [16], and real data corresponding to the MBRs of 556,696 census blocks (polygons) of Iowa, Kansa, Missouri and Nebraska, provided by Tiger [6]. For comparison purpose the number of synthetic objects, generated following a uniform or a skewed distribution, is also set to 556,696 . The capacity of each server is set to 2,000 objects.

\subsection{Cost of the redistribution}

A first experiment is performed to stress the impact of the maximal pivot height allowed for the data redistribution.

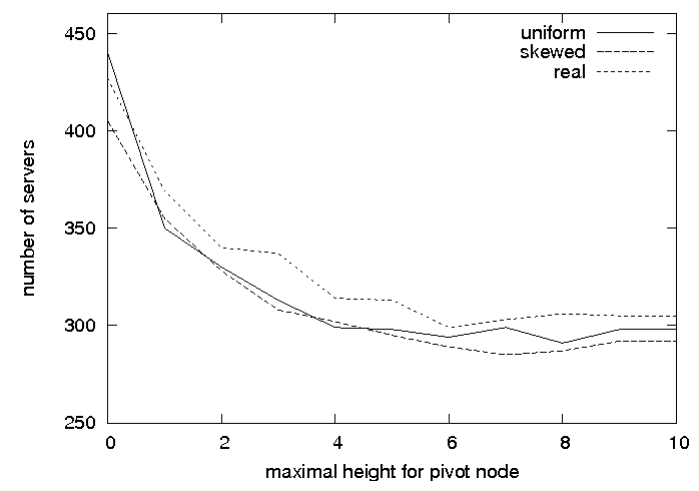

(a) Number of servers

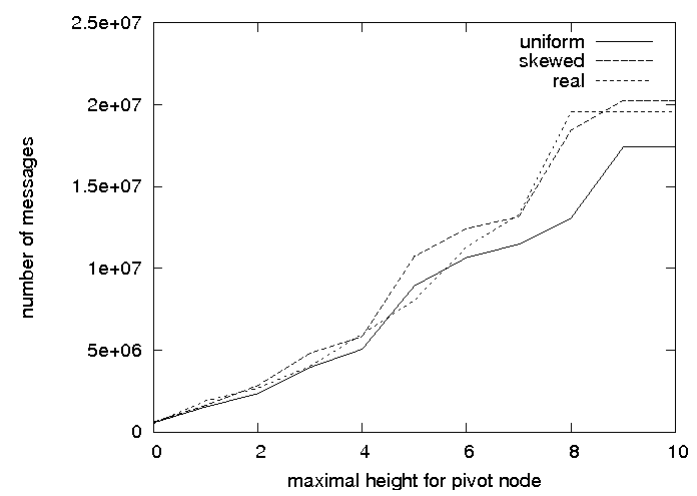

(b) Number of messages

Figure 10: Impact of the maximal height for the pivot node

First note that with our setting, whatever the distribution is, the height of the tree is 10. Figure 10a shows that our redistribution algorithm highly reduces the number of servers requested, even for small values of the maximal height $h_{p}$ of a pivot node. For instance with a uniform distribution, the number of servers without redistribution is 440 . With $h_{p}$ set to 1 , this number falls to 350 , so a gain of $21 \%$ of the required resources. With higher values for $h_{p}$, the number of servers can reach 291 , so a gain of $34 \%$. We observe similar results with other distributions.

The skewed distribution leads to a lower number of servers if we do not use redistribution, compared to other datasets. The reason is that insertions are concentrated on a specific part of the indexing area, hence concerns mostly a subset of the pool. These nodes fill in up to their capacity, then split and, since they still cover the dense insertion area, remain subject to high insertions load. With uniform distribution, each node newly created is initially half-empty, and its probability to receive new insertion requests is similar to that of the other nodes.t This leads to a lower average space occupancy $(63 \%)$ than with skewed data (69\%), and therefore so a higher number of servers (Figure 11).

Using the redistribution algorithm, one achieves a high fill-in rate for the servers, i.e. up to $96 \%$ with uniform distribution, $98 \%$ with skewed distribution, and $93 \%$ with real data (Figure 11). This value is already reached with a medium value of $h_{p}$ like 4 or 5 . With $h_{p}$ set to 1 , the improvement is still noteworthy, e.g., $79 \%, 78 \%$ and $75 \%$ for respectively uniform, skewed and real datasets.

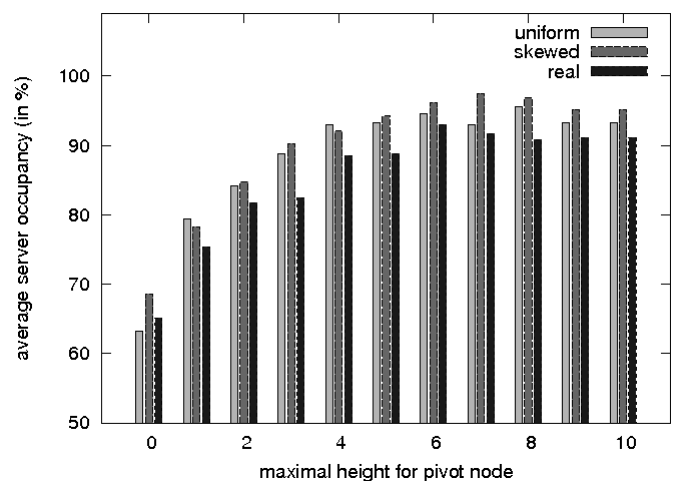

Figure 11: Average server's occupancy rate w.r.t. maximal pivot's height

Figure 10b shows the cost in number of messages of the redistribution strategy. Depending on the distribution, a rebalancing with a maximal pivot's height set to 1 requires between 2 and 4 times more messages. If we allow the pivot node to be at any height, possibly up to the root, the number of messages reaches a value 30 times higher with our data! Indeed, with this complete flexibility, the tree is almost full and a new insertion generally leads to a costly iterative redistribution process, that may affect all the nodes of the tree in the worst case. Analysis of figures 10a-b suggests that setting $h_{p}$ to 4 provides generally a number of servers very close to the best possible space occupancy, with a number of messages "only" 10 times higher than without redistribution.

\subsection{Analysis of server allocation profiles}

The second set of experiments illustrates how our solution may be deployed in architectures supporting a mixed strategy. We make now the practical assumption that the "traditional" insertion mechanism (without redistribution) is used when there are servers available. If, at some point, the system lacks of storage resource, it dynamically switches to the redistribution mode, until new servers are added. We call "server allocation profile" the set of parameters that describe this evolution, including the initial size of the server pool, the average time necessary to extend the pool, and the number of servers added during an extension.

The experiment assumes that the system consists initially of 200 servers. When new resources are requested, a set of additional 25 servers is allocated. The shortage period 
between the request for new servers and their effective allocation (represented in our profile by the number of insertion requests) corresponds to a forced redistribution mode and constitutes the variable parameter that we analyse. We measure the behaviour of the system as the total number of servers and messages required, the maximal height for the pivot node being set to 1,2 or 3 .

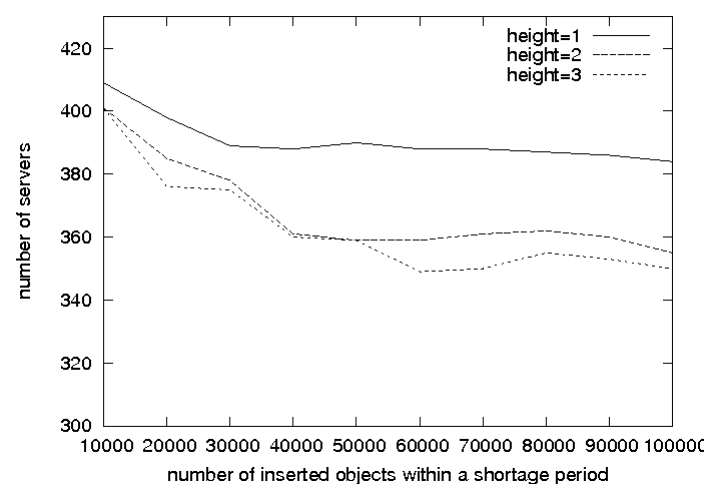

(a) Number of servers

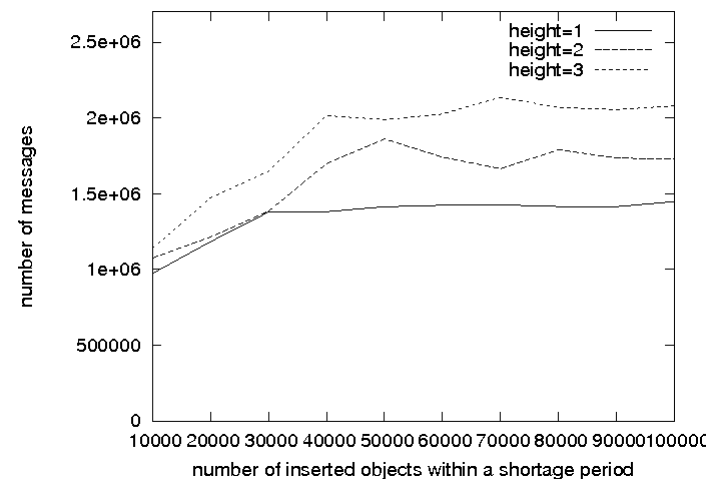

(b) Number of messages

Figure 12: Impact of the number of inserted objects during a shortage period

As expected, the higher the allowed height for the pivot node is, the lower is the number of requested servers (Figure 12.a). Obviously, the impact is opposite on the number of messages (Figure 12.b). Both figures show that the system can handle a shortage of servers during a period corresponding to up to 100,000 insertions with a limited cost (here at most 3 times the cost without shortage). The decreasing aspect of the curves in Figure 12a is due to the storage balancing effect: with a long shortage period, many objects are inserted and they trigger a redistribution, thereby optimizing servers capacity. The amount of storage balancing increases, and so does the total number of messages.

\section{CONCLUSION}

Our framework enables a Rtree-based indexing structure for large spatial data sets stored over interconnected servers. We present a $k$-NN querying algorithm and a data distribution algorithm that limits the number of servers and improve the storage utilization, at the cost of additional messages. We believe that the scheme should fit the needs of new applications using endlessly larger sets of spatial data.

\section{REFERENCES}

[1] N. Beckmann, H. Kriegel, R. Schneider, and B. Seeger. The $\mathrm{R}^{*}$ tree : An Efficient and Robust Access Method for Points and Rectangles. In Proc. ACM Symp. on the Management of Data (SIGMOD), pages 322-331, 1990.

[2] A. Crainiceanu, P. Linga, J. Gehrke, and J. Shanmugasundaram. Querying Peer-to-Peer Networks Using P-Trees. In Proc. Intl. Workshop on the Web and Databases (WebDB), pages 25-30, 2004

[3] R. Devine. Design and Implementation of DDH: A Distributed Dynamic Hashing Algorithm. In Foundations of Data Organization and Algorithms (FODO), 1993.

[4] C. du Mouza, W. Litwin, and P. Rigaux. SD-Rtre: A Scalable Distributed Rtree. In Proc. Intl. Conf. on Data Engineering (ICDE), pages 296-305, 2007.

[5] V. Gaede and O. Guenther. Multidimensional Access Methods. ACM Computing Surveys, 30(2), 1998.

[6] U. C. B. Geography Division. Tiger/Line files, 2007. URL:http://www.census.gov/geo/www/tiger/.

[7] A. Guttman. R-trees : A Dynamic Index Structure for Spatial Searching. In Proc. ACM Symp. on the Management of Data (SIGMOD), pages 45-57, 1984.

[8] H. Jagadish, B. C. Ooi, and Q. H. Vu. BATON: A Balanced Tree Structure for Peer-to-Peer Networks. In Proc. Intl. Conf. on Very Large Data Bases (VLDB), pages 661-672, 2005.

[9] H. Jagadish, B. C. Ooi, Q. H. Vu, R. Zhang, and A. Zhou. VBI-Tree: A Peer-to-Peer Framework for Supporting Multi-Dimensional Indexing Schemes. In Proc. Intl. Conf. on Data Engineering (ICDE), 2006.

[10] J. S. Karlsson. hQT*: A Scalable Distributed Data Structure for High-Performance Spatial Accesses. In Foundations of Data Organization and Algorithms (FODO), 1998.

[11] V. Kriakov, A. Delis, and G. Kollios. Management of Highly Dynamic Multidimensional Data in a Cluster of Workstations. In Proc. Intl. Conf. on Extending Data Base Technology (EDBT), pages 748-764, 2004.

[12] W. Litwin, M.-A. Neimat, and D. A. Schneider. RP*: A Family of Order Preserving Scalable Distributed Data Structures. In Proc. Intl. Conf. on Very Large Data Bases (VLDB), pages 342-353, 1994.

[13] W. Litwin, M.-A. Neimat, and D. A. Schneider. LH* A Scalable, Distributed Data Structure. ACM Trans. on Database Systems, 21(4):480-525, 1996.

[14] N. Roussopoulos, S. Kelley, and F. Vincent. Nearest Neighbor Queries. In Proc. ACM Symp. on the Management of Data (SIGMOD), 1995.

[15] H. Samet. Foundations of Multi-dimensional Data Structures. Morgan Kaufmann Publishing, 2006.

[16] Y. Theodoridis, J. R. O. Silva, and M. A. Nascimento. On the Generation of Spatiotemporal Datasets. In Proc. Intl. Conf. on Large Spatial Databases (SSD), 1999. 